REGARDS

SUR LEECONOMIE ALLEMANDE

BULLETIN ECONOMIQUE DU CRAC

\section{Regards sur l'économie allemande}

Bulletin économique du CIRAC

$102 \mid 2011$

Varia

\title{
L'arrêt de Karlsruhe sur les aides budgétaires dans la zone euro
}

\section{Peter-Christian Müller-Graff}

Traducteur : Isabelle Bourgeois

\section{OpenEdition}

Journals

\section{Édition électronique}

URL : http://journals.openedition.org/rea/4322

DOI : $10.4000 /$ rea. 4322

ISBN : 978-2-8218-1134-8

ISSN : 1965-0787

Éditeur

CIRAC

Édition imprimée

Date de publication : 31 octobre 2011

Pagination : 5-14

ISSN : 1156-8992

\section{Référence électronique}

Peter-Christian Müller-Graff, «L'arrêt de Karlsruhe sur les aides budgétaires dans la zone euro », Regards sur l'économie allemande [En ligne], 102 I octobre 2011, mis en ligne le 01 octobre 2013, consulté le 06 mai 2019. URL : http://journals.openedition.org/rea/4322 ; DOI : 10.4000/rea.4322 


\section{L'arrêt de Karlsruhe sur les aides budgétaires dans la zone Euro}

\author{
Peter-Christian Müller-Graff
}

L'arrêt rendu le 7 septembre 2011 par le Tribunal constitutionnel fédéral (2 BvR 987/10, 2 BvR 1485/10, 2 BvR 1099/10) examine la conformité avec la Loi fondamentale de la législation allemande en matière d'intégration européenne, plus précisément celle des deux lois d'aide budgétaire au sein de la zone Euro votées en mai 2010. Il s'agit d'une part de la Loi du 7 mai 2010 relative à la prise en charge de garanties pour la préservation de la solvabilité de la République Hellénique, nécessaire à la stabilité financière au sein de l'Union monétaire (Gesetz zur Übernahme von Gewährleistungen zum Erhalt der für die Finanzstabilität in der Währungsunion erforderlichen Zahlungsfähigkeit der Hellenischen Republik), surnommée Loi sur la stabilité financière dans I'UEM (Währungsunion-Finanzstabilitätsgesetz, WFStG), et d'autre part de la Loi du 22 mai 2010 sur la prise en charge de garanties dans le cadre d'un mécanisme européen de stabilité (Gesetz zur Übernahme von Gewährleistungen im Rahmen eines europäischen Stabilisierungsmechanismus).

L'arrêt considère que ces deux lois sont conformes à la Constitution (alinéa 119). Néanmoins, son argumentation soulève une fois de plus un certain nombre de questions quant à l'impact de ce mécanisme sur la politique européenne de l'Allemagne et au rôle du Tribunal constitutionel fédéral dans le cadre de la politique d'intégration européenne. Ces questions avaient déjà été évoquées dans l'arrêt du 12 décembre 1993 sur les lois d'approbation du Traité de Maastricht (2 BVR 2134/92, 2 BVR 2159/92- BVerfGE 89, 155), la décision du 31 mars 1998 sur l'introduction de l'Euro (BVR 1877/94, BVerfGE 97, 350), et l'arrêt du 30 juin 2009 sur la loi d'approbation du Traité de Lisbonne (BvE 2/08 et al., BVerfGE 123, 267). Il convient donc d'évaluer le dernier arrêt de la Cour de Karlsruhe à l'aune de sa jurisprudence précédente.

Nous commencerons ainsi par présenter ses lignes directrices (I) avant d'examiner les éléments nouveaux qu'il apporte au cadre constitutionnel dans lequel évoluent la politique européenne et la politique de transferts de l'Allemagne (II), le rôle à venir des pouvoirs exécutif, législatif et judiciaire dans sa politique de transferts au sein de la zone Euro (III), et les éventuelles protections juridiques s'offrant aux citoyens allemands contre les aides budgétaires dans la zone Euro (IV).

\section{I - Les lignes directrices de l'arrêt du 7 septembre 2011}

Dans les lignes directrices du chapitre présentant les conclusions (al. 93-141), le Tribunal se livre à une analyse différenciée de la recevabilité du recours en constitutionnalité (1), développe le principe démocratique sous l'angle de l'autonomie budgétaire et de l'interdiction de l'automatisme budgétaire (2), présente les considérants sur la conformité avec la Constitution des deux lois de soutien financier de mai 2010 (3), souligne que ce qui légitime la participation de l'Allemagne à l'Union monétaire est le caractère de communauté de stabilité que lui confère le droit européen (4) et exprime quelques considérations générales sur les préalables de droit constitutionnel que devra respecter à l'avenir la politique allemande en matière d'aides financières au sein de l'UEM (5).

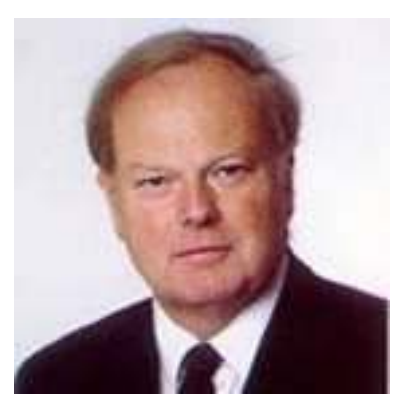

Prof. P.-C. Müller-Graff, Directeur de l'Institut für deutsches und europäisches Gesellschafts- und Wirtschaftsrecht, Université de Heidelberg 
Motif du recours : atteinte au droit de vote

Le lien entre droit de vote et autonomie budgétaire du parlement

Les griefs irrecevables
L'analyse différenciée de la recevabilité de la plainte en constitutionnalité (recours individuel)

L'arrêt traite d'abord avec exhaustivité la question de la recevabilité des recours en constitutionnalité contre ces lois, formulées à titre individuel par plusieurs personnes, qui sont à l'origine de la procédure de contrôle (al. 93-119). La Loi fondamentale ouvre en effet le droit à chaque citoyen de déposer un recours constitutionnel s'il s'estime lésé par la puissance publique dans l'un de ses droits fondamentaux (la Loi fondamentale les énumère dans ses 19 premiers articles) ou un droit assimilé (Art. 93, § 1, al. 4a de la Loi fondamentale, LF).

Les plaignants avaient mis en avant le fait que ces lois de soutien lésaient leur droit subjectif de participation à l'élection des députés au Bundestag (Art. 38, § 1 et $\S 2$ LF), étant donné que ces mesures d'aide destinées à d'autres Etats membres porteraient atteinte au caractère durable de l'autonomie budgétaire du Bundestag. La Cour considère comme recevable le motif incriminé - la réduction de l'autonomie budgétaire du Bundestag (al. 93) - et examine donc le bien fondé du recours comme de ses motifs (al. 120 sq.). La recevabilité se justifie (al. 95 sq.) par le fait que l'exposé des plaignants comporte suffisamment d'arguments probants pour démontrer qu'ils sont personnellement et directement lésés dans leur droit de vote (art. $38 \mathrm{LF}$ ).

Ce lien entre droit de vote et autonomie budgétaire du parlement peut surprendre un lecteur français et demande donc à être explicité. Dans cet arrêt, le Tribunal constitutionnel fédéral confirme l'approche développée dans sa jurisprudence précédente (al. 98) et récuse expressément les critiques formulées par une partie des sciences juridiques (al. 101). II souligne à plusieurs reprises que le droit de vote ne peut être réduit à un acte formel de légitimation de la puissance publique à l'échelon fédéral, mais que, du fait que l'acte matériel de vote relève fondamentalement de l'essence de la démocratie, celui-ci vise aussi à garantir l'exercice effectif de la souveraineté du peuple. Et il en conclut que, de ce fait, le droit de vote protège le citoyen-électeur contre toute perte de substance de ce pouvoir par " des transferts étendus ou ... substantiels » de missions et compétences du Bundestag à des institutions supranationales ou par le biais " d'engagements comparables contractés avec d'autres Etats..., et qui présentent un lien institutionnel avec l'Union supranationale ", Iorsque ceux-ci "limitent durablement l'auto-gouvernement démocratique du peuple au point qu'il ne lui est plus possible de prendre des décisions politiques cruciales de façon autonome » (voir al. 98, 100 et 103 sq.).

La raison invoquée par la Cour est celle-ci : "le droit du citoyen à la démocratie, qui repose foncièrement sur la dignité de l'Homme, ... se trouverait invalidé si le parlement abandonnait des éléments essentiels de l'autodétermination politique et retirait ainsi durablement au peuple son pouvoir d'influence démocratique ».

Son arrêt limite certes les possibilités de recours en la matière au grief de " modifications structurelles dans l'architecture juridique de l'organisation de l'Etat " (al. 100), mais en y incluant les délégations de pouvoir par le législateur fédéral en application d'accords de droit international, " susceptibles, de par leur nature et leur ampleur, de porter massivement atteinte à l'autonomie budgétaire » (al. 103). II fonde cette interprétation sur la considération que la prise de décision sur les recettes et les dépenses de la puissance publique est consubstantielle des droits parlementaires en démocratie et que ceux-ci ne peuvent donc pas, en violation de la démocratie, être vidés de leur substance "par entravement du législateur budgétaire » (al. 104). Or les engagements contractés par la République fédérale dans le cadre d'accords internationaux visant à préserver la liquidité d'autres Etats membres de l'UEM pourraient présenter un tel potentiel.

A l'inverse, l'arrêt considère comme irrecevables les autres griefs avancé par les plaignants, puisés dans le débat public. Ils estimaient, premièrement, leur droit de vote lésé également par le fait que, par leur impact, les lois incriminées 
s'assimileraient à " une modification, non fixée par traités », de la finalité du droit primaire de l'Union européenne, à savoir garantir la stabilité des prix au sein de la zone Euro (motifs insuffisamment étayés) ; Ils s'estimaient, deuxièmement, lésés dans leur droit à la propriété (art. 14 LF) par les risques inflationnistes (baisse du pouvoir d'achat) inhérents aux mesures d'aide (motifs insuffisamment étayés ; al. 110 sq.). Leur troisième grief portait sur la participation du gouvernement fédéral aux actions de soutien prises à l'échelon européen le 10 mai 2010 (absence de plainte directe déposée ; al. 115), leur quatrième sur les mesures de soutien conclues par le Conseil de l'UE et les chefs de gouvernement le 10 mai 2010, ainsi que sur l'achat d'obligations émises par la Grèce et d'autres Etats de la zone Euro par la Banque Centrale Européenne (ces mesures ne sont pas un acte de souveraineté de la RFA et ne peuvent donc donner lieu à recours ; al. 116) ; le cinquième enfin sur le fait que la Commission européenne et le gouvernement fédéral auraient omis de prendre en mai 2010 des mesures pour éviter la crise (motifs insuffisamment étayés ; al. 117 sq.).

Développement du principe de démocratie sous l'angle de l'autonomie budgétaire et de l'interdiction de l'automatisme budgétaire

A propos des lois de soutien allemandes, l'arrêt précise ensuite les conséquences du principe de démocratie inhérent au droit de vote garanti par la Loi fondamentale sur les décisions de la puissance publique en matière de recettes et de dépenses (al. 120 sq.). Trois assertions-clés les concrétisent. La Cour considère premièrement que le droit de vote est lésé "si le Bundestag se dessaisit de sa responsabilité budgétaire parlementaire de telle sorte que ni lui ni ses successeurs ne seront en mesure d'exercer leur pouvoir budgétaire en pleine responsabilité » (al. 121). Deuxièmement, le Bundestag n'est pas habilité à transférer son pouvoir budgétaire à d'autres acteurs en leur conférant des mandats de politique budgétaire indéterminés (al. 125). Troisièmement, la Cour déclare que, quand elle prend acte d'un tel abandon prohibé de l'autonomie budgétaire, elle se limite aux "violations manifestes » en ce qui concerne le volume financier et qu'elle respecte ce faisant la latitude d'appréciation du législateur (al. 130).

La première assertion définit concrètement la portée du principe de démocratie en matière de décisions budgétaires : le Bundestag doit "conserver le contrôle sur ses choix budgétaires fondamentaux, même dans une système de gouvernance intergouvernemental ». II convient de relever à cet égard que la Cour ajoute que le principe de démocratie n'interdit nullement au Bundestag de se lier "substantiellement" par sa politique financière dans le cadre de l'intégration européenne. Mais il exige que le Bundestag "reste le lieu où se prennent en pleine autonomie les décisions en matière de recettes et de dépenses » (al. 124).

La seconde assertion définit plus concrètement "l'interdiction de se dessaisir de la responsabilité budgétaire " faite au Bundestag. II signifie que celui-ci ne doit notamment pas "s'exposer à des mécanismes affectant les finances... et susceptibles d'entraîner des charges pour le budget sans son approbation constitutive préalable » (al. 125). C'est là un prolongement de l'arrêt rendu à propos du Traité de Lisbonne. La Cour souligne en effet que le principe de démocratie se trouverait violé par le transfert d'éléments essentiels du pouvoir budgétaire du Bundestag « si la détermination du type comme du montant des prélèvements frappant le citoyen était substantiellement supra-nationalisée » (al. 126). Et elle énonce formellement que la Loi fondamentale exige donc "que le législateur budgétaire prenne ses décisions sur les recettes et dépenses sans être déterminé dans ses choix par les organes et les autres Etats membres de l'Union européenne, et qu'il demeure durablement 'maître de ses propres décisons'».

La Loi fondamentale ferme donc la voie à une union financière européenne supranationale ; pour cela, il faudrait que l'Allemagne adopte une nouvelle Consti-

\section{Le Bundestag}

ne peut pas se dessaisir

de son pouvoir budgétaire

Même dans I'UE, il doit rester maître de ses choix ...

... et donc refuser tout automatisme 
La Cour limite son contrôle aux violations manifestes

Le gouvernement fédéral doit recueilli

l'approbation préalable du Bundestag tution. Or la Cour considère que, déjà, la préservation de la solvabilité d'autres Etats membres présente " une situation de tension importante ». Elle conclut en conséquence " qu'il est interdit au Bundestag d'approuver tout automatisme en matière de cautions ou de versements décidé à l'échelon intergouvernemental ou supranational, non assorti de strictes conditions et non limité dans ses effets, et qui, une fois déclenché, échapperait à son contrôle et à son influence » (al. 127). En toute logique, deux interdits en découlent : d'une part celui de conférer tout mandat global pour la prise de garanties financières au bénéfice des choix budgétaires et fiscaux d'un autre Etat membre ; d'autre part, celui de prendre part à tout mécanisme adopté dans le cadre de traités internationaux « qui auraient pour conséquence la prise en charge de la responsabilité décisionnelle d'autres Etats » (al. 1128).

Avec la troisième assertion, la Cour réduit l'étendue de l'examen de l'affaire qu'elle avait à connaître : la question de savoir si le Bundestag respecte cette interdiction de se dessaisir de son autonomie budgétaire. Elle limite son contrôle aux violations " manifestes » lorsqu'elle cherche à déterminer " jusqu'à quel montant le législateur budgétaire peut engager sa responsabilité avec une prise de garantie, en considération de l'ouverture de risques et de leurs conséquences à attendre quant à son autonomie " (al. 131). L'arrêt considère toutefois qu'il lui semble "improbable » de parvenir à fixer par la jurisprudence un plafond au volume de garanties.

\section{La constitutionnalité des lois de soutien financier adoptées en mai 2010}

Dans leur arrêt, les juges de Karlsruhe considèrent enfin que les deux lois de soutien financier adoptées en mai 2010 sont conformes avec la Loi fondamentale (al. 133-141). Ils sont très succincts dans leurs considérants. Ils répondent par la négative à la question du franchissement d'un plafond - qu'ils ont l'intelligence de ne pas chiffrer - de charges à ne pas dépasser. Et ils avancent deux raisons : d'abord, en cas de responsabilité engagée, ces deux lois n'auraient assurément pas pour effet " que, dans la pratique, l'autonomie budgétaire... serait entièrement vidée de son sens pendant une durée considérable »(al. $135)$; ensuite, aucune des deux lois ne créerait ou ne consoliderait un automatisme menant le Bundestag à se dessaisir de son pouvoir budgétaire (al. 136).

Relevons ici que ces considérants s'appuient d'une part sur le constat que les bases juridiques au fondement de l'Union économique et monétaire n'autorisent pas un tel automatisme. C'est là un avertissement aux Etats de la zone Euro, aux organes politiques de l'UE et à la BCE, leur intimant de ne pas déroger aux dispositions prévues par le Traité sur le fonctionnement de l'UE (TFUE). Dans le débat public allemand, en effet, la conformité avec ce traité des mesures adoptées en mai 2010 et, nommément, l'achat par la BCE d'obligations émises par des Etats membres en difficulté, est pour le moins controversée. D'autre part, les considérants s'appuient sur le constat que les deux lois de soutien ne comportent aucune disposition annihilant durablement le pouvoir budgétaire du Bundestag, étant donné que la finalité, le montant et les modalités du mandat afférent sont clairement définies (al. 138 sq.). Toutefois, la Cour considère comme insuffisant le devoir fait au Bund de "s'efforcer" seulement de recueillir " l'accord » de la Commission des finances du Bundestag avant d'engager sa responsabilité par la Loi sur la prise en charge de garanties dans le cadre d'un mécanisme européen de stabilité. Au contraire, le principe de démocratie fait obligation au gouvernement fédéral de recueillir l'approbation préalable de la Commission des finances.

Ce qui légitime la participation de l'Allemagne à l'Union monétaire est le caractère de communauté de stabilité que lui confère le droit européen

Le contrôle en constitutionnalité des deux lois s'appuie sur un présupposé de droit européen. Celui-ci est évoqué sous deux aspects. 
Premièrement, l'arrêt souligne que le TFUE conçoit l'Union monétaire comme une communauté de stabilité et que l'autonomie budgétaire nationale est constitutive de l'organisation actuelle de I'UEM. Dans l'approche du Tribunal constitutionnel fédéral, cet élément était déjà essentiel pour le contrôle en constitutionnalité de la Loi d'approbation du Traité de Maastricht (arrêt du 12 octobre 1993, 2 BvR 2134/92 et 2 BvR 2159/92). Le présent arrêt répète donc : "la conception contractuelle de I'UEM comme une communauté de stabilité constitue le fondement et l'objet de la loi d'approbation allemande » (al. 129).

Et il considère que, dans le droit communautaire, des normes centrales du TFUE sur l'UEM garantissent l'exigence de préservation du principe de démocratie inscrit dans la Loi fondamentale, qui est la base légitimant l'intégration de la RFA dans l'UEM.

Ces normes sont l'objectif prioritaire de la stabilité des prix (art. 127 TFUE) et l'indépendance de la BCE (art. 130 TFUE); elles répondent aux exigences de la Loi fondamentale : indépendance de la BCE et stabilité des prix (art. 88, § 2 LF) et garantie du droit à la propriété (art. 14 LF). Il en va de même de l'interdiction faite à la BCE d'acquérir directement des titres de créances d'organismes publics (art. 123 TFUE), de celle faite à un Etat de répondre des engagements des autres Etats membres (art. 125 TFUE) et des dispositions relatives aux critères de stabilité s'appliquant à la politique budgétaire des Etats membres (art. 126, 136 TFUE).

L'arrêt justifie la constitutionnalité des deux lois d'application en s'appuyant également sur son interprétation du cadre réglementaire de I'UEM, à savoir que celui-ci s'oppose à tout automatisme par lequel le Bundestag serait amené à se dessaisir de son pouvoir budgétaire (al. 137). Cette argumentation prolonge l'attendu de l'arrêt sur le Traité de Maastricht, selon lequel l'évolution de l'UEM est établie sur des normes prévisibles par ce traité et préserve ainsi la responsabilité des parlements. Cet état de fait garantit que l'Allemagne "ne puisse pas être prise dans l'engrenage de l'automatisme d'une communauté de responsabilité solidaire aux effets imprévisibles car échappant à tout contrôle du fait de sa dynamique autonome ".

La conception même du Traité fait donc que les conséquences des deux lois d'approbation restent prévisibles. Elles présupposent en effet également le respect des engagements contractuels par tous les acteurs. Ce présupposé semble toutefois quelque peu idéaliste au vu du comportement peu conforme aux traités d'une BCE se livrant tout récemment à l'acquisition d'obligations d'Etats en proie à d'importants problèmes budgétaires.

\section{Les considérations générales sur les préalables de droit constitutionnel que devra respecter à l'avenir la politique allemande en matière d'aides financières au sein de I'UEM}

Les considérations générales nombreuses dont est assorti le jugement appellent une attention particulière. D'une part, elles établissent des règles pour l'action du gouvernement fédéral et du législateur (Bundestag). D'autre part, en en référant de façon récurrente à ce qu'il est convenu d'appeler la " clause d'intangibilité » (Ewigkeitsklausel) de la Loi fondamentale (art. 79, § 3 LF), l'arrêt fait allusion aux limites d'une modification de la Constitution. Cette clause interdit en effet toute modification de la Loi fondamentale qui toucherait à l'essence des principes énoncés aux art. 1 et 20 LF, à savoir le respect de la dignité humaine, le fait que le pouvoir législatif, de même que les pouvoirs exécutif et judiciaire sont liés par l'ordre constitutionnel et les droits fondamentaux, et enfin les principes qui fondent la structure de l'Etat (dont le principe de démocratie).

Ces considérations tout particulièrement doivent être analysées dans la perspective de la nouvelle approche qu'elles sont susceptibles d'apporter aux principes constitutionnels liant la politique européenne et la politique de transferts de l'Allemagne.
L'autonomie budgétaire nationale est constitutive de I'UEM...

... et le TFUE préserve le principe de démocratie

L'UEM est établie sur des normes prévisibles

Intangibilité de la Loi fondamentale 


\section{II - Nouvelle approche des principes constitutionnels liant la politique européenne et la politique de transferts de la RFA}

La nouvelle approche développée par cet arrêt d'une part prolonge les lignes directrices de l'arrêt rendu à propos du Traité de Lisbonne (1), et d'autre part concrétise la souveraineté budgétaire du Bundestag en énonçant une série de règles (2).

\section{Continuité des lignes directrices : le principe de démocratie}

Les lignes directrices de cet arrêt sont inscrites dans la continuité de l'arrêt rendu à propos du Traité de Lisbonne (voir Müller-Graff, REA 92/2009). Ses attendus reposent sur le principe de démocratie (art. 20, § 1 LF), en liaison avec la dignité humaine (art. $1 \mathrm{LF}$ ) et le caractère irrévocable de ces principes constitutionnels (art. 79, $\S 3 \mathrm{LF}$ ). Toutefois, l'argumentation ne répond pas toujours clairement à la question de savoir s'il est possible de faire évoluer l'organisation concrète du principe de démocratie par une modification de la Constitution ou si celui-ci échappe foncièrement à toute modification.

Dans la continuité de la jurisprudence de la Cour s'inscrit ainsi expressément la justification d'un recours en constitutionnalité dans le cas où le grief porte sur une violation du droit de vote (élection des députés au Bundestag) induite par l'annihilation des pouvoirs du Bundestag dans le cadre d'un transfert de compétences effectué par des traités internationaux (al. 100). Et dans le même temps, l'arrêt confirme que la norme constitutionnelle fondamentale en la matière est que soient garantis le plein exercice du pouvoir du peuple et donc, à un degré suffisant, les pouvoirs substantiels du Bundestag (al. 98, 120).

\section{Concrétisation du principe de démocratie en ce qui concerne l'autonomie budgétaire du Bundestag}

La nouvelle interprétation du droit constitutionnel concerne l'extension du domaine d'application du principe de démocratie à la souveraineté budgétaire du Bundestag et, ainsi, à la politique de transferts de la République fédérale dans I'Union monétaire. C'est là l'argument au cœur de cet arrêt.

II rappelle que, déjà, l'arrêt rendu à propos du Traité de Lisbonne déclarait que le pouvoir budgétaire est un élément fondamental pour l'auto-détermination démocratique de l'Etat (al. 122). Et il en conclut en toute logique à l'argument clé évoqué ci-dessus, à savoir que, en qualité de représentants du peuple, les députés du Bundestag doivent conserver le contrôle des décisions fondamentales en matière de politique budgétaire, y compris dans un système de gouvernance intergouvernemental (al. 124).

Les engagements internationaux comportent un risque

Or le Tribunal considère que les mandats conférés à l'exécutif pour mettre en œuvre ses engagements internationaux destinés à préserver la liquidité d'autres Etats de l'UE comportent un potentiel de risque particulier pour l'autonomie décisionnelle du Bundestag (al. 105). II en tire une série de conséquences en ce qui concerne les rôles respectifs des pouvoirs législatif, exécutif et judiciaire dans la cadre de la politique de transferts allemande au sein de la zone Euro.

Concrétisation du rôle des pouvoirs législatif, exécutif et judiciaire dans la cadre de la politique allemande de transferts au sein de la zone Euro

Rôle du Bundestag

Le gouvernement doit solliciter l'approbation de la Commission des Finances
Le Bundestag est le premier concerné. Si l'arrêt renforce son rôle vis-à-vis du gouvernement fédéral, il lui pose aussi certaines limites.

Le renforcement des pouvoirs du Bundestag est particulièrement net dans les attendus relatifs à la Loi sur la stabilité financière dans I'UEM (Euro-Stabilisierung-Gesetz) de mai 2010. En effet, le Tribunal estime que le principe de démocratie, en l'occurrence sous la forme du pouvoir budgétaire parlementaire, 
est insuffisamment respecté lorsque le gouvernement fédéral se contente de "s'efforcer " d'obtenir l'accord de la Commission des Finances du Bundestag avant de prendre l'engagement international d'apporter sa garantie financière, ainsi que le prévoit cette loi. II considère au contraire que cette obligation doit " subir une interprétation " conforme à la Loi fondamentale en ce sens qu'il " est du devoir [du gouvernement fédéral] de solliciter l'approbation préalable de la Commission des Finances " (al. 141). Si le doute est permis quant à la nature juridique de cette interprétation de la loi (" devoir de solliciter " l'approbation au lieu de "s'efforcer » d'obtenir l'accord du Bundestag), du moins le message est clair. Le droit constitutionnel interdit à l'exécutif de prendre quelque garantie financière que ce soit, pour un autre Etat membre ou dans le cadre d'un fonds d'aide international, sans l'approbation préalable de la Commission des Finances (voir ci-dessous les implications pratiques pour le gouvernement fédéral).

Les pouvoirs du Bundestag sont par ailleurs élargis, mais ce renforcement est évoqué de manière hautement abstraite : il concerne les " grands engagements à venir ", mais sans que ceux-ci soient quantifiés. Ainsi, "toute mesure de soutien solidaire du Bund dans le contexte international ou dans celui de l'Union et impliquant des dépenses budgétaires d'une certaine ampleur » doit être approuvée "de manière isolée ". Cela vaut tout particulièrement pour les accords supranationaux "qui, du fait des sommes engagées, sont susceptibles d'avoir un impact structurel sur le pouvoir budgétaire » (al. 128). L'arrêt cite en exemple l'octroi de garanties "dont l'exécution peut mettre en danger l'autonomie budgétaire ", ou encore "la participation à des systèmes de garanties financières afférents ». Dans ce cas, estime le Tribunal, il découle du principe de démocratie que, premièrement, " chaque mesure isolée " requiert l'approbation du Bundestag et que, deuxièmement, il faut veiller "à ce que soit conservée une influence parlementaire suffisante sur la manière dont sont utilisés les moyens ainsi mis à disposition $»$.

A l'évidence, les critères de "l'ampleur » et de la " mise en danger de l'autonomie budgétaire » restent très flous. De même, le Tribunal n'explique pas s'il est prêt à étendre également cette " influence parlementaire » au Parlement européen ; mais compte tenu de la logique argumentative développée à propos de l'autonomie budgétaire du Bundestag, on peut affirmer que le Tribunal y est par principe opposé.

Par ailleurs, l'arrêt évoque à plusieurs reprises des limites constitutionnelles générales pour les mesures de transferts financiers adoptés par le Bundestag. Mais il n'est pas toujours explicite sur la question de savoir si ces limites concernent 'seulement' la législation normale ou s'il ne s'agit pas aussi de limites 'éternelles' que pose la Loi fondamentale à la législation modifiant la Constitution (art. 79, § 3 LF).

Parmi les limites imposées au législateur normal, le Tribunal rappelle les règles renforcées pour le recours au crédit du Bund et des Länder ('règle d'or'), adoptées récemment par modification de la Loi fondamentale (al. 104). A juste titre, il reconnaît dans cette obligation et "limitation sensible » de la latitude d'action des parlements - paradoxales en apparence seulement - une visée plus profonde : celle d'asseoir durablement leur capacité de façonner la démocratie. L'actualité révèle certes les conséquences dramatiques d'un endettement disproportionné. Mais le Tribunal estime qu'il est "douteux » de faire découler du principe de démocratie la fixation, par la jurisprudence, d'un plafond pour les transferts budgétaires et laisse, comme nous l'avons vu, cette question en suspens (al. 131).

Enfin, le Tribunal définit comme limite à la latitude d'action du Bundestag - une limite qu'il n'est vraisemblablement pas possible de surmonter, même par une modification de la Constitution - le concept abstrait de " l'interdiction de se dessaisir de la responsabilité budgétaire ». Appliqué au contexte européen, il signi-
A fortiori pour les " grands engagements à venir »

Une approche à préciser

Imprécision sur la nature des imites du pouvoir parlementaire

Celles qui s'imposent au législateur normal

Les limites imposées par la Constitution 
Rôle du gouvernement fédéral

Il est dans l'intérêt du gouvernement de consulter la Commission des Finances

« Grands » engagements : approbation constitutive du Bundestag

Rôle du Tribunal constitutionnel fédéral

Ne pouvant intervenir dans l'action politique 'normale', ... fie l'interdiction "de transférer la responsabilité budgétaire à d'autres acteurs... par des délégations non définies de pouvoir en matière de politique budgétaire ». A titre d'exemples, l'arrêt nomme : la participation à des mécanismes "pouvant mener à des charges budgétaires incommensurables sans que celles-ci aient donné lieu à approbation constitutive préalable » (al. 125); la délégation à l'échelon supranational de décisions quant au type et au montant de prélèvements lorsque ceux-ci frappent le citoyen "dans une grande ampleur » (al. 126) ; l'approbation du Bundestag à une " décision imposée de l'extérieur [Fremdbestimmung] par les organes et d'autres Etats membres de I'UE " (interdite au demeurant, même par une modification de la Loi fondamentale) ; l'approbation d'un automatisme de garanties et de transferts transnational si celle-ci n'est pas soumise à de strictes conditions et limitée dans ses effets; enfin, l'autorisation générale et forfaitaire de la prise de garanties "d'une ampleur importante » (al. 127).

Quoi qu'il en soit, le Tribunal estime que la Loi fondamentale interdit « de faire peser sur les budgets du Bund, présents ou à venir, des engagements d'un montant disproportionné, même s'il ne s'agit que de cautions », et que cet interdit n'est vraisemblablement pas abrogeable (al. 133).

Ce renforcement des pouvoirs constitutionnels du Bundestag a pour corollaire de lier le gouvernement fédéral au vote du Bundestag lors de la prise de garanties destinées à d'autres Etats membres ou à tout fonds de soutien international, quel qu'il soit.

L'exigence de l'approbation préalable de la Commission des Finances (al. 141) ne devrait pas se révéler un grand handicap dans la pratique, puisque sa composition reflète la majorité du Bundestag et que cette majorité porte le gouvernement fédéral. II est donc peu probable que la majorité de la Commission des Finances fasse échouer un projet de transfert proposé par un gouvernement fédéral porté par la majorité du Bundestag. Or il est dans l'intérêt même du gouvernement fédéral de consulter au préalable les membres de la Commission des Finances.

Dans la pratique, le lien soumettant les décisions du gouvernement fédéral au vote du Bundestag sera renforcé, puisque ce n'est pas la seule Commission des Finances qui devra donner son approbation, mais l'ensemble du Bundestag (al. 128). C'est le cas des " grands engagements » (al. 128). Une autre mesure que le gouvernement ne peut prendre "sans l'approbation constitutive du Bundestag " est la prise de garanties "d'importante ampleur..., menant à la communautarisation directe ou indirecte de dettes souveraines, ... et où le déclenchement de la mise en œuvre de garanties dépendrait donc exclusivement du comportement d'autres Etats " (al. 105). Le droit constitutionnel s'opposerait donc à la création d'obligations européennes (ou Euro-Bonds) sans l'approbation du Bundestag.

Soulignons enfin que le Tribunal constitutionnel fédéral a eu l'intelligence de limiter expressément son propre rôle dans l'appréciation de la politique de transferts de la République fédérale au sein de la zone Euro. Certes, la concrétisation des règles constitutionnelles que doivent respecter le gouvernement fédéral et le Bundestag impliquent également que le Tribunal constitutionnel fédéral décide, dans le cas d'une saisine, de l'interprétation à donner à ces critères comme de leur l'application.

Néanmoins, il prévient le risque de se voir entraîné dans l'action politique 'normale' et d'y prendre une part de responsabilité dans les choix effectués en matière de politique de transfert et leurs conséquences pour le budget allemand. [NDT : Rappelons ici que la Cour suprême allemande se distingue de son homologue américaine en ce sens que son statut lui interdit de prendre position dans le débat politique (pas de "political question »; voir REA 52-53/2001)]. Car une telle prise de position entrerait en contradiction avec la notion classique de la séparation des pouvoirs au sens où l'entendait Montesquieu, mais aussi 
avec celle qu'établit la Loi fondamentale entre le pouvoir judiciaire et le domaine de compétence des organes politiques.

En toute logique, lorsque le Tribunal constate que, au vu du montant des engagements financiers internationaux, le Bundestag se dessaisit de son autonomie budgétaire (ce qui lui est interdit), il limite donc sa mission aux cas de "violations manifestes " (al. 131). Ce faisant, il confirme avec une netteté bienvenue qu'il se doit de respecter la marge d'appréciation du législateur, démocratiquement appelé à cette fonction; et il rapporte cette prérogative législative tout particulièrement à l'évaluation du risque de devoir déclencher la mise en œuvre des garantieset à celle de la soutenabilité future du budget du Bund comme de la compétitivité économique de la République fédérale (al. 132).

Il convient de relever en outre que, à la différence de celui rendu à propos du Traité de Lisbonne, le présent arrêt n'évoque à aucun moment la question des liens hypothétiques entre le Tribunal constitutionnel fédéral et les organes de I'Union, y compris la Cour européenne de Justice. II est vrai que rien ne l'imposait non plus.

\section{IV - Protections juridiques éventuelles s'offrant aux citoyens allemands contre les aides budgétaires}

Soulignons enfin que le Tribunal se réserve le droit de trancher ultérieurement la question de l'éventualité d'une protection juridique individuelle contre les aides budgétaires de l'Etat allemand au sein de la zone Euro. II prolonge ainsi dans le domaine budgétaire sa jurisprudence, développée dans l'arrêt à propos du Traité de Maastricht, sur l'étendue des recours en constitutionnalité. Elle se fonde sur l'argument selon lequel toute mesure prise par la puissance publique allemande dans le contexte de l'intégration européenne ouvre droit à recours au motif qu'elle vide de son contenu le droit de vote pour les élections au Bundestag. En ce qui concerne les aides budgétaires au sein de la zone Euro, le pivot est dès lors la préservation de la substance même de l'autonomie budgétaire du Bundestag. L'arrêt rappelle également à plusieurs reprises la notion de limites infranchissables que pose la Loi fondamentale à une révision de la Constitution.

En revanche, à l'inverse de celui rendu à propos du Traité de Lisbonne, l'arrêt ne thématise pas expressément le droit à protection juridique ouvert en dernière extrémité contre les actes hors constitutionnalité (" ultra-vires ») des organes de l'Union. Si on développe cette approche, alors on peut imaginer que cela pourrait s'appliquer également, le cas échéant, aux résolutions de soutien financier du Conseil européen ou à l'acquisition directe d'obligations d'Etat par la BCE. Dans le même temps, l'arrêt ne se prononce pas sur la question de savoir si un recours en constitutionnalité serait recevable s'il porte sur des mesures prises hors traité par la puissance publique allemande mais qui influent sur le droit communautaire primaire ou le complètent (al. 109).

Cela étant, le Tribunal constitutionnel fédéral limite nettement son rôle en matière de contrôle en constitutionnalité de la politique d'aides budgétaires au sein de la zone Euro: d'abord par l'auto-limitation de ses compétences, du fait de l'ampleur des cautions accordées, aux violations manifestes; ensuite par le respect de la latitude d'appréciation du législateur en ce qui concerne le risque d'avoir à s'acquitter d'une caution; enfin par le renoncement à fixer une limite supérieure contraignante pour la prise de garanties.

NÉANMOINS, CES GRANDS JALONS DE DROIT CONSTITUTIONNEL IMPLIQUENT qu'il faudra s'attendre, à l'avenir aussi, au dépôt de recours individuels ; ceux-ci amèneront le Tribunal constitutionnel fédéral à se prononcer sur les préalables et les limites fixés par la Loi fondamentale à la politique allemande en matière de transferts dans la zone Euro. De la sorte, le Tribunal confirme son rôle d'ins-
... il limite sa mission aux cas de " violation manifeste »

Pivot :

le droit de vote

L'arrêt ne se prononce pas sur les constats ultra-vires

Auto-limitation de la Cour en matière d'aides budgétaires 
tance potentielle de réflexion sur la compatibilité entre les mesures de soutien adoptées au sein de la zone Euro avec la souveraineté de la République fédérale, et poursuit ainsi sa prise de participation au débat de politique européenne.

Sous l'angle du droit comparé, on peut se demander si une Cour française, en l'occurrence le Conseil constitutionnel, serait susceptible d'adopter une position similaire à propos des actes des organes de l'Etat engageant les finances publiques (voir à ce propos Müller-Graff/Reichel, 1998 ; Müller-Graff/Lenk, 2001 ; Müller-Graff, 2011). Cette question soulève également celle du poids respectif des obligations constitutionnelles auxquelles se trouvent liées les mesures politiques adoptées dans ce contexte en Allemagne et en France.

(Traduction : I. Bourgeois)

\section{Indications bibliographiques}

- Bourgeols I., « La constitutionnalisation de l'ordre économique. Les 50 ans du Bundesverfassungsgericht », Regards sur l'économie allemande, n 92, juillet 2009

-EverLING U., "Wirtschaftspolitik und Finanzhilfe in der Europäischen Union », in MüLLER-GRAFF P.-C., Schmahl S., Skouris V. (eds), Europäisches Recht in Bewährung und Wandel. Festschrift für Dieter H. Scheuing, Baden-Baden, 2011

- Götz V., « Das Maastricht-Urteil des Bundesverfassungsgerichts », JZ, 1993

- HÄBERLE P., «Das retroperspektive Lissabon-Urteil als versteinernde Maastricht-II-Entscheidung », JöR, vol. 58, 2010

- LÜBBE-WoLfF G., "Europäisches und nationales Verfassungsrecht », in Veröffentlichungen der Vereinigung der Deutschen Staatsrechtslehrer, vol. 60, 2001

- MüLleR-GRAFF P.-C., « Das Karlsruher Lissabon-Urteil : Bedingungen, Grenzen, Orakel und integrative Optionen », integration, 2009

- Müller-Graff P.-C., « L'arrêt de Karlsruhe sur le Traité de Lisbonne », Regards sur l'économie allemande, $\mathrm{n}^{\circ}$ 92, juillet 2009

- MülleR-Graff P.-C., LeNK J., « Rahmenbedingungen des deutschen Verfassungsrechts für die Mitwirkung an der EU - Kontinuität oder Wandel ? », in SchNeIDER H., Jopp M., Schmalz U. (eds), Eine neue deutsche Europapolitik ?, 2001

- MüLleR-Graff P.-C., Reichel A., « Die europäische Integration aus der Sicht der Rechtsprechung nationaler Verfassungsgerichte ", in JOPP M., MAURER A., SCHNEIDER H. (eds), Europapolitische Grundverständnisse im Wandel, 1998

- SCHEUING D. H., « Deutsches Verfassungsrecht und europäische Integration », in MüLLER-GRAFF P.-C., RITZER C. (eds), Europäisches öffentliches Recht - Ausgewählte Beiträge, Baden-Baden, 2006

- SCheuINg D. H., « Europäisierung als Zielsetzung und Herausforderung des deutschen Verfassungsrechts ", in MüLleR-GRAfF P.-C., RITZER C. (eds), Europäisches öffentliches Recht - Ausgewählte Beiträge, Baden-Baden, 2006

- SCHRÖDER M., « Das Bundesverfassungsgericht als Hüter des Staates in der europäischen Integration », DVBI., 1994

- SeIDEL M., « Eine Wirtschaftsregierung für die Europäische Union - rechtliche Grundlagen, Legitimation, Funktion und Verhältnis zur Europäischen Zentralbank ", in MüLLER-GRAFF P.-C., SchmaHL S., SkouRIS V. (eds), Europäisches Recht in Bewährung und Wandel. Festschrift für Dieter H. Scheuing, Baden-Baden, 2011

- Tomuschat C., « Die Europäische Union unter der Aufsicht des Bundesverfassungsgerichts », EUGRZ, 1993

- www.bundesverfassungsgericht.de. 